PROCEEDINGS OF THE

AMERICAN MATHEMATICAL SOCIETY

Volume 136, Number 12, December 2008, Pages 4185-4195

S 0002-9939(08)09438-0

Article electronically published on July 23, 2008

\title{
ON THE COHEN-MACAULAYNESS OF FIBER CONES
}

\author{
DUONG QUÔC VIỆT
}

(Communicated by Bernd Ulrich)

\begin{abstract}
Let $A$ be a Noetherian local ring with the maximal ideal $\mathfrak{m}$ and $I$ an ideal of $A$. Denote by $F_{A}(I)=\bigoplus_{n>0}\left(I^{n} / \mathfrak{m} I^{n}\right)$ the fiber cone of $I$. This paper characterizes the multiplicity and the Cohen-Macaulayness of fiber cones in terms of minimal reductions of ideals.
\end{abstract}

\section{INTRODUCTION}

Throughout this paper, let $(A, \mathfrak{m})$ be a Noetherian local ring with maximal ideal $\mathfrak{m}$, infinite residue field $k=A / \mathfrak{m}$, and Krull dimension $\operatorname{dim} A=d>0$. Define $F_{A}(I)=\bigoplus_{n>0}\left(I^{n} / \mathfrak{m} I^{n}\right)$ to be the fiber cone of an ideal $I$ in $A$. Denote by $\ell(I)=\operatorname{dim} F_{A}(I)$ the analytic spread of $I$, by $\mu(I)$ the minimum number of generators of $I$, by $r=r_{J}(I)$ the reduction number of $I$ with respect to a minimal reduction $J$, and by $v(A)=\mu(\mathfrak{m})$ the embedding dimension of $A$. In recent years, the Cohen-Macaulayness and other properties of special fiber rings have attracted much attention (see for instance [C-G-P-U], C-P-V2], Co-Z], D-R-V], [H-H], J-V-V1, [J-P-V]).

Using different approaches, some authors gave criteria for the Cohen-Macaulayness of fiber cones. For instance, Huneke and Sally $[\mathrm{H}-\mathrm{S}]$ proved that if $A$ is CohenMacaulay and $I$ is m-primary with $r(I) \leqslant 1$, then $F_{A}(I)$ is Cohen-Macaulay. More generally, Shah Sh1, Sh2 showed the sufficient condition that $F_{A}(I)$ is CohenMacaulay if $I$ is an equimultiple ideal with grade $I=$ ht $I$ and $r(I) \leqslant 1$. Under the assumption that a minimal reduction $J$ of $I$ is generated by a regular sequence and $J \cap I^{n}=J I^{n-1}$ for all $n \leqslant r_{J}(I)$, Cortadellas and Zarzuela [C-Z] proved that the Cohen-Macaulayness of $F_{A}(I)$ is equivalent to $J \cap \mathfrak{m} I^{n}=J \mathfrak{m} I^{n-1}$ for all $n \leqslant r_{J}(I)$.

This paper characterizes the multiplicity (Theorem 2.3, Section 2) and the Cohen-Macaulayness (Theorem 3.1, Section 3) of fiber cones in terms of minimal reductions of ideals. As interesting consequences of main results, we get e.g. a generalization of results of Huneke-Sally and Shah [op. cit.] (Proposition 3.2, Section 3). In the case that minimal reductions of $I$ are generated by a regular sequence, we get a result that seems to account well for the essence of the theorem of Cortadellas-Zarzuela [op. cit.] (see Corollary 3.5, Section 3).

Received by the editors May 15, 2006, and, in revised form, November 3, 2007, and November 13, 2007.

2000 Mathematics Subject Classification. Primary 13H10; Secondary 13A15, 13A30, 13C14, $13 \mathrm{H} 15$.

Key words and phrases. Cohen-Macaulay ring, multiplicity, fiber cone.

(C)2008 American Mathematical Society 
A ring $A$ is said to have minimal multiplicity if $e(A)=v(A)-d+1$. It is also known by Sally [Sa] that $A$ is Cohen-Macaulay with minimal multiplicity if and only if $A$ is Cohen-Macaulay and $r(\mathfrak{m}) \leqslant 1$. By using the main results, we answer the question as to when the fiber cone is Cohen-Macaulay with minimal multiplicity (Theorem 3.6, Section 3).

This paper is divided into three sections. Section 2 investigates the multiplicity of fiber cones. Section 3 is devoted to the discussion of the Cohen-Macaulayness of fiber cones.

\section{The MUlTiPlicity of FIBER CONES}

Using the concept of weak-(FC)-sequences in Vi1, this section presents some results on the multiplicity of the fiber cone.

We first review some results and notions. Set

$$
\mathfrak{a}: \mathfrak{b}^{\infty}=\left\{x \in A \mid \text { there is a positive integer } n \text { such that } x \mathfrak{b}^{n} \subseteq \mathfrak{a}\right\} .
$$

Definition (Vi] $)$. Let $I$ be a non-nilpotent ideal of $A$. Set $A^{*}=A / 0: I^{\infty}$, $\mathfrak{m}^{*}=\mathfrak{m} A^{*}$, and $I^{*}=I A^{*}$. Recall that an element $x \in I\left(x^{*}\right.$ is the image of $x$ in $\left.A^{*}\right)$ is called a weak-(FC)-element with respect to $(\mathfrak{m}, I)$ if there exists an integer $n_{1}$ such that

$\left(\mathrm{FC}_{1}\right): x \in I \backslash \mathfrak{m} I$, and for all $n \geq n_{1}$ and for all non-negative integers $m$,

$$
\mathfrak{m}^{* m} I^{* n} \cap\left(x^{*}\right)=\mathfrak{m}^{* m} I^{* n-1} x^{*} .
$$

$\left(\mathrm{FC}_{2}\right): x$ is a filter-regular element with respect to $I$, i.e., $0: x \subseteq 0: I^{\infty}$.

Let $x_{1}, x_{2}, \ldots, x_{t}$ be a sequence in $I$. For each $i=0,1, \ldots, t-1$, set $A_{i}=$ $A /\left(x_{1}, x_{2}, \ldots, x_{i}\right) ; \bar{x}_{i+1}$ is the image of $x_{i+1}$ in $A_{i}, I_{i}=I A_{i}$, and $\mathfrak{m}_{i}=\mathfrak{m} A_{i}$. The sequence $x_{1}, \ldots, x_{t}$ is called a weak-(FC)-sequence in $I$ with respect to $(\mathfrak{m}, I)$ if $\bar{x}_{i+1}$ is a weak-(FC)-element with respect to $\left(\mathfrak{m}_{i}, I_{i}\right)$ for each $i=0,1, \ldots, t-1$.

An ideal $J$ is called a reduction of ideal $I$ if $J \subseteq I$ and $I^{n+1}=J I^{n}$ for some $n$. A reduction $J$ is called a minimal reduction of $I$ if it does not properly contain any other reduction of $I[\mathrm{~N}-\mathrm{R}]$. The least integer $n$ such that $I^{n+1}=J I^{n}$ is called the reduction number of $I$ with respect to $J$, and we denote it by $r_{J}(I)$. The reduction number of $I$ is defined by $r(I)=\min \left\{r_{J}(I) \mid J\right.$ is a minimal reduction of $\left.I\right\}$.

By using the Rees lemma in $\mathrm{Re}$, the author $\mathrm{Vi2}-\mathrm{Vi3}$ showed that if $I$ is non-nilpotent, the length of any maximal weak-(FC)-sequence in $I$ with respect to $(\mathfrak{m}, I)$ is equal to $\ell(I)$, and if $x_{1}, x_{2}, \ldots, x_{\ell}$ is a weak-(FC)-sequence in $I$ with respect to $(\mathfrak{m}, I)$, then $\left(x_{1}, x_{2}, \ldots, x_{\ell}\right)$ is a minimal reduction of $I$. Moreover, as in the proof of Theorem 3.3 of [Vi4], then any minimal reduction of $I$ is generated by a maximal weak-(FC)-sequence in $I$ with respect to $(\mathfrak{m}, I)$. So we have:

Note 1 . An ideal $J \subseteq I$ is a minimal reduction of $I$ if and only if $J$ is generated by a weak-(FC)-sequence of length $\ell(I)$ in $I$ with respect to $(\mathfrak{m}, I)$.

The following proposition plays an important role in the proofs of this paper.

Proposition 2.1. Let $I$ be an ideal with analytic spread $\ell(I)=\ell>0$. Let $x_{1}, x_{2}, \ldots$, $x_{\ell}$ be a weak-(FC)-sequence in I with respect to $(\mathfrak{m}, I)$. Set $J=\left(x_{1}, x_{2}, \ldots, x_{\ell}\right)$. For any $i<\ell$, set

$$
A_{i}=A /\left(x_{1}, \ldots, x_{i}\right), I_{i}=I A_{i}, P_{i}=\left(x_{1}, x_{2}, \ldots, x_{i}\right): I^{\infty}, A_{i}^{\prime}=A / P_{i}, I_{i}^{\prime}=I A_{i}^{\prime} .
$$


Then

(i) $e\left(F_{A}(I)\right)=e\left(F_{A_{i}}\left(I_{i}\right)\right)=e\left(F_{A_{i}^{\prime}}\left(I_{i}^{\prime}\right)\right)$.

(ii) $l\left[\frac{F_{A_{i}^{\prime}}\left(I_{i}^{\prime}\right)}{J F_{A_{i}^{\prime}}\left(I_{i}^{\prime}\right)}\right] \leqslant l\left[\frac{F_{A_{i}}\left(I_{i}\right)}{J F_{A_{i}}\left(I_{i}\right)}\right] \leqslant l\left[\frac{F_{A}(I)}{J F_{A}(I)}\right]$.

(iii) $l\left[\frac{F_{A_{i}^{\prime}}\left(I_{i}^{\prime}\right)}{J F_{A_{i}^{\prime}}\left(I_{i}^{\prime}\right)}\right]=l\left[\frac{F_{A}(I)}{J F_{A}(I)}\right]$ if and only if $I^{n} \cap P_{i} \subseteq J I^{n-1}\left(\bmod \mathfrak{m} I^{n}\right)$ for all $1 \leqslant$ $n \leqslant r_{J}(I)$.

Proof. Set $N=0: I^{\infty}, A^{*}=A / N, \mathfrak{m}^{*}=\mathfrak{m} A^{*}, I^{*}=I A^{*}$. By the Artin-Rees lemma, there exists an integer $u$ such that $N \cap I^{n}=I^{n-u}\left(N \cap I^{u}\right) \subseteq I^{n-u} N$ for all $n \geq u$. Since $I^{n-u} N=0$ for all large enough $n, N \cap I^{n}=0$ for all large enough $n$. From this it follows that

$$
l_{A^{*}}\left(\frac{I^{* n}}{\mathfrak{m}^{*} I^{* n}}\right)=l_{A}\left(\frac{I^{n}+N}{\mathfrak{m} I^{n}+N}\right)=l_{A}\left(\frac{I^{n}}{\mathfrak{m} I^{n}+N \cap I^{n}}\right)=l_{A}\left(\frac{I^{n}}{\mathfrak{m} I^{n}}\right)
$$

for all large enough $n$. Hence

$$
e\left(F_{A}(I)\right)=e\left(F_{A^{*}}\left(I^{*}\right)\right) .
$$

Let $x \in I$ be a weak-(FC)-element with respect to $(\mathfrak{m}, I)$. If $x^{*}$ is the image of $x$ in $A^{*}$, then $x^{*}$ is not contained in any prime ideal belonging to Ass $A^{*}$ (see condition $\left.\left(\mathrm{FC}_{2}\right)\right)$. Hence $x^{*}$ is a non-zero divisor in $A^{*}$. Set $B=A^{*} / x^{*} A^{*}, \mathfrak{b}=\mathfrak{m}^{*} B, \mathfrak{c}=I^{*} B$. For all large enough $n$, we have

$$
\begin{aligned}
l_{B}\left(\frac{\mathfrak{c}^{n}}{\mathfrak{b c}^{n}}\right) & =l_{A^{*}}\left(\frac{I^{* n}+\left(x^{*}\right)}{\mathfrak{m}^{*} I^{* n}+\left(x^{*}\right)}\right)=l_{A^{*}}\left(\frac{I^{* n}}{\mathfrak{m}^{*} I^{*^{n}}+\left(x^{*}\right) \cap I^{* n}}\right) \\
& =l_{A^{*}}\left(\frac{I^{* n}}{\mathfrak{m}^{*} I^{* n}+x^{*} I^{* n-1}}\right)\left(\text { see condition }\left(\mathrm{FC}_{1}\right)\right) \\
& =l_{A^{*}}\left(\frac{I^{* n}}{\mathfrak{m}^{*} I^{* n}}\right)-l_{A^{*}}\left(\frac{\mathfrak{m}^{*} I^{* n}+x^{*} I^{* n-1}}{\mathfrak{m}^{*} I^{* n}}\right) \\
& =l_{A^{*}}\left(\frac{I^{* n}}{\mathfrak{m}^{*} I^{* n}}\right)-l_{A^{*}}\left(\frac{x^{*} I^{* n-1}}{\mathfrak{m}^{*} I^{*^{n}} \cap\left(x^{*}\right) \cap I^{* n}}\right) \\
& =l_{A^{*}}\left(\frac{I^{* n}}{\mathfrak{m}^{*} I^{* n}}\right)-l_{A^{*}}\left(\frac{x^{*} I^{* n-1}}{x^{*} \mathfrak{m}^{*} I^{* n-1}}\right) .
\end{aligned}
$$

Since $x^{*}$ is a non-zero divisor in $A^{*}$, it follows that $l_{A^{*}}\left(\frac{I^{* n-1}}{\mathfrak{m}^{*} I^{* n-1}}\right)=l_{A^{*}}\left(\frac{x^{*} I^{* n-1}}{x^{*} \mathfrak{m}^{*} I^{* n-1}}\right)$, so that

$$
l_{B}\left(\frac{\mathfrak{c}^{n}}{\mathfrak{b c}^{n}}\right)=l_{A^{*}}\left(\frac{I^{* n}}{\mathfrak{m}^{*} I^{* n}}\right)-l_{A^{*}}\left(\frac{I^{* n-1}}{\mathfrak{m}^{*} I^{* n-1}}\right)
$$

for all large enough $n$. Hence if $\ell>1$, then

$$
e\left(F_{A^{*}}\left(I^{*}\right)\right)=e\left(F_{B}(\mathfrak{c})\right) .
$$

The case of $\ell(I)>1, \mathfrak{c}$ is non-nilpotent in $B$. Set $B^{\prime}=B / 0: \mathfrak{c}^{\infty}$ and $\mathfrak{c}^{\prime}=\mathfrak{c} B^{\prime}$. Then by (1) we get that

$$
e\left(F_{B}(\mathfrak{c})\right)=e\left(F_{B^{\prime}}\left(\mathfrak{c}^{\prime}\right)\right) .
$$

Set $A_{1}=A /(x), I_{1}=I A_{1}, A_{1}^{\prime}=A /(x): I^{\infty}$, and $I_{1}^{\prime}=I A_{1}^{\prime}$. Note that $B^{\prime} \cong$ $A /(x): I^{\infty}=A_{1}^{\prime}$. Then by $(1)$, we have

$$
e\left(F_{A_{1}}\left(I_{1}\right)\right)=e\left(F_{A_{1}^{\prime}}\left(I_{1}^{\prime}\right)\right)=e\left(F_{B^{\prime}}\left(\mathfrak{c}^{\prime}\right)\right) .
$$

Combining (4) with (3), (2) and (1), we get

$$
e\left(F_{A}(I)\right)=e\left(F_{A_{1}}\left(I_{1}\right)\right)=e\left(F_{A_{1}^{\prime}}\left(I_{1}^{\prime}\right)\right) .
$$


Assume that the analytic spread $\ell=\ell(I)>1$ and $x_{1}, x_{2}, \ldots, x_{i}(i<\ell)$ is a weak(FC)-sequence in $I$ with respect to $(\mathfrak{m}, I)$. Set $A_{i}=A /\left(x_{1}, \ldots, x_{i}\right), I_{i}=I A_{i}, A_{i}^{\prime}=$ $A /\left(x_{1}, \ldots, x_{i}\right): I^{\infty}$, and $I_{i}^{\prime}=I A_{i}^{\prime}$. Then using (5) and by induction on $i<\ell=\ell(I)$, we easily show that $e\left(F_{A}(I)\right)=e\left(F_{A_{i}}\left(I_{i}\right)\right)=e\left(F_{A_{i}^{\prime}}\left(I_{i}^{\prime}\right)\right)$. Note that this equation is true too in the case of $\ell=1$ by (1). This establishes (i).

Assume that $r_{J}(I)=r$. Set $Q_{i}=\left(x_{1}, \ldots, x_{i}\right), P_{i}=Q_{i}: I^{\infty}$. It can be verified that

$$
\begin{aligned}
l\left[F_{A}(I) / J F_{A}(I)\right] & =1+\sum_{1 \leqslant n \leqslant r} l\left[I^{n} /\left(J I^{n-1}+\mathfrak{m} I^{n}\right)\right], \\
l\left[F_{A_{i}}\left(I_{i}\right) / J F_{A_{i}}\left(I_{i}\right)\right] & =1+\sum_{1 \leqslant n \leqslant r} l\left[Q_{i}+I^{n} /\left(Q_{i}+J I^{n-1}+\mathfrak{m} I^{n}\right)\right] \\
& =1+\sum_{1 \leqslant n \leqslant r} l\left[I^{n} /\left(Q_{i} \cap I^{n}+J I^{n-1}+\mathfrak{m} I^{n}\right)\right], \\
l\left[F_{A_{i}^{\prime}}\left(I_{i}^{\prime}\right) / J F_{A_{i}^{\prime}}\left(I_{i}^{\prime}\right)\right] & =1+\sum_{1 \leqslant n \leqslant r} l\left[P_{i}+I^{n} /\left(P_{i}+J I^{n-1}+\mathfrak{m} I^{n}\right)\right] \\
& =1+\sum_{1 \leqslant n \leqslant r} l\left[I^{n} /\left(P_{i} \cap I^{n}+J I^{n-1}+\mathfrak{m} I^{n}\right)\right] .
\end{aligned}
$$

It is clear that

$$
\left(J I^{n-1}+\mathfrak{m} I^{n}\right) \subseteq\left(Q_{i} \cap I^{n}+J I^{n-1}+\mathfrak{m} I^{n}\right) \subseteq\left(P_{i} \cap I^{n}+J I^{n-1}+\mathfrak{m} I^{n}\right)
$$

for all $1 \leqslant n \leqslant r_{J}(I)$. Hence we immediately get (ii) and (iii).

Recall that $\mu(I)$ is the minimum number of generators of $I$ and

$$
\mu(I)=\operatorname{dim}_{k}(I / \mathfrak{m} I)=l(I / \mathfrak{m} I) .
$$

Note 2. It can be easily seen that if $a$ is a non-zero divisor in $A$, then $\mu(a I)=\mu(I)$.

At this point we remark that: On the one hand, by Proposition 2.1 we have $e\left(F_{A}(I)\right)=e\left(F_{A_{\ell-1}}\left(I_{\ell-1}\right)\right)$. On the other hand, since $J=\left(x_{1}, x_{2}, \ldots, x_{\ell}\right)$ is a minimal reduction of $I$, the analytic spread of $I_{\ell-1}$ is 1 .

Thus the problem is reduced to the case of the fiber cone of an ideal with analytic spread $\ell=1$. This is the reason why we need the following lemma.

Lemma 2.2. Let $I$ be an ideal with analytic spread $\ell(I)=1$ and $x$ a weak-(FC)element in $I$ with respect to $(\mathfrak{m}, I)$. Set $r_{x A}(I)=r$ and $I^{0}=A$. Then

(i) $e\left(F_{A}(I)\right)=\mu\left(I^{n}\right)$ for all $n \geq r$ if grade $I=1$.

(ii) $e\left(F_{A}(I)\right)=\mu\left(\frac{I^{n}+0: I^{\infty}}{0: I^{\infty}}\right)$ for all $n \geq r$.

(iii) $e\left(F_{A}(I)\right) \leqslant \mu\left(I^{r}\right)$ with equality if and only if $\left(0: I^{\infty}\right) \cap I^{r}=\left(0: I^{\infty}\right) \cap \mathfrak{m} I^{r}$.

Proof. Since $\ell(I)=1, l\left(I^{n} / \mathfrak{m} I^{n}\right)=\mu\left(I^{n}\right)$ takes a constant value for all large enough $n$. From this it follows that $e\left(F_{A}(I)\right)=\mu\left(I^{n}\right)$ for all large enough $n$. Remember that $r_{(x)}(I)=r$. Hence $I^{n}=I^{r} x^{n-r}$ for all $n>r$. Now, if grade $I>0$, then $x^{n-r}$ is a non-zero divisor in $A$. By Note 2 we have $\mu\left(I^{n}\right)=\mu\left(I^{r} x^{n-r}\right)=\mu\left(I^{r}\right)$ for all $n \geq r$. We get (i).

Set $A^{*}=A / 0: I^{\infty}$ and $I^{*}=I A^{*}$. We recall that by Proposition 2.1 we have $e\left(F_{A}(I)\right)=e\left(F_{A^{*}}\left(I^{*}\right)\right)$. Since $\left(0: I^{\infty}\right): I=0: I^{\infty}$, it follows that $\operatorname{grade}_{A^{*}} I^{*}>0$. Hence by (i) we get

$$
e\left(F_{A}(I)\right)=e\left(F_{A^{*}}\left(I^{*}\right)\right)=\mu\left(I^{* r}\right)=\mu\left(\frac{I^{r}+0: I^{\infty}}{0: I^{\infty}}\right) .
$$


This completes the proof of (ii).

It is easily seen that

$$
\begin{aligned}
\mu\left(\frac{I^{r}+0: I^{\infty}}{0: I^{\infty}}\right) & =\mu\left(\frac{I^{r}}{I^{r} \cap\left(0: I^{\infty}\right)}\right) \\
& =l_{A}\left(\frac{I^{r}}{\mathfrak{m} I^{r}}\right)-l_{A}\left(\frac{I^{r} \cap\left(0: I^{\infty}\right)+\mathfrak{m} I^{r}}{\mathfrak{m} I^{r}}\right) \\
& =\mu\left(I^{r}\right)-l_{A}\left(\frac{I^{r} \cap\left(0: I^{\infty}\right)}{\mathfrak{m} I^{r} \cap I^{r} \cap\left(0: I^{\infty}\right)}\right) \\
& =\mu\left(I^{r}\right)-l_{A}\left(\frac{I^{r} \cap\left(0: I^{\infty}\right)}{\mathfrak{m} I^{r} \cap\left(0: I^{\infty}\right)}\right) .
\end{aligned}
$$

Hence by (ii), $e\left(F_{A}(I)\right) \leqslant \mu\left(I^{r}\right)$ with equality if and only if $\left(0: I^{\infty}\right) \cap I^{r}=(0$ : $\left.I^{\infty}\right) \cap \mathfrak{m} I^{r}$. Thus, we get (iii).

By combining Proposition 2.1 with Lemma 2.2, we obtain the following theorem.

Theorem 2.3. Let $(A, \mathfrak{m})$ be a Noetherian local ring with maximal ideal $\mathfrak{m}$ and infinite residue field $k=A / \mathfrak{m}$. Let $I$ be an ideal with analytic spread $\ell(I)=\ell>0$ and $J$ a minimal reduction of $I$. Suppose that $J$ is generated by $x_{1}, x_{2}, \ldots, x_{\ell}, a$ weak-(FC)-sequence in $I$ with respect to $(\mathfrak{m}, I)$. Set $J=\left(x_{1}, x_{2}, \ldots, x_{\ell}\right), r_{J}(I)=r$, and $Q=\left(x_{1}, x_{2}, \ldots, x_{\ell-1}\right)$. Then

(i) $e\left(F_{A}(I)\right)=\mu\left(\frac{I^{n}+Q: I^{\infty}}{Q: I^{\infty}}\right)$ for all $n \geq r$.

(ii) $e\left(F_{A}(I)\right) \leqslant \mu\left[\left(I^{r}+Q\right) / Q\right]$ with equality if and only if

$$
\left(Q: I^{\infty}\right) \cap I^{r}=\left(Q: I^{\infty}\right) \cap \mathfrak{m} I^{r}(\bmod Q) .
$$

(iii) $e\left(F_{A}(I)\right)=\mu\left[\left(I^{r}+Q\right) / Q\right]$ if $\left(Q: I^{\infty}\right) \cap I^{r} \subseteq Q$.

(iv) $e\left(F_{A}(I)\right) \leqslant \mu\left(I^{r}\right)-\ell(I)+1$.

Proof. Set $A_{\ell-1}=A / Q, I_{\ell-1}=I A_{\ell-1}, A_{\ell-1}^{\prime}=A / Q: I^{\infty}$, and $I_{\ell-1}^{\prime}=I A_{\ell-1}^{\prime}$. Then by Proposition 2.1 we have $e\left(F_{A}(I)\right)=e\left(F_{A_{\ell-1}}\left(I_{\ell-1}\right)\right)$, since $\left(x_{1}, x_{2}, \ldots, x_{\ell}\right)$ is a minimal reduction of $I$. Hence $\ell\left(I_{\ell-1}\right)=1$. Lemma 2.2(ii) immediately gives $e\left(F_{A_{\ell-1}}\left(I_{\ell-1}\right)\right)=\mu\left(I_{\ell-1}^{\prime n}\right)$ for all $n \geq r$. Thus, we get (i).

By Lemma 2.2(iii), $e\left(F_{A_{\ell-1}}\left(I_{\ell-1}\right)\right) \leqslant \mu\left(I_{\ell-1}^{r}\right)$, since $e\left(F_{A}(I)\right)=e\left(F_{A_{\ell-1}}\left(I_{\ell-1}\right)\right)$ and

$$
\mu\left(I_{\ell-1}^{r}\right)=\mu\left[\left(I^{r}+Q\right) / Q\right]
$$

it follows that $e\left(F_{A}(I)\right) \leqslant \mu\left[\left(I^{r}+Q\right) / Q\right]$, since $e\left(F_{A}(I)\right)=\mu\left[\left(I^{r}+Q\right) / Q\right]$ if and only if

$$
e\left(F_{A_{\ell-1}}\left(I_{\ell-1}\right)\right)=\mu\left(I_{\ell-1}^{r}\right) .
$$

Hence by Lemma 2.2(iii) we get (ii).

Since $\left(Q: I^{\infty}\right) \cap I^{r} \subseteq Q$, it follows that $Q+\left(Q: I^{\infty}\right) \cap I^{r}=Q=Q+(Q$ : $\left.I^{\infty}\right) \cap \mathfrak{m} I^{r}$. Hence by (ii) we have (iii).

Set $\mu\left(I^{r}\right)=s$. Since $\left(x_{1}, x_{2}, \ldots, x_{\ell}\right)$ is a minimal reduction of $I,\left(x_{1}^{r}, x_{2}^{r}, \ldots, x_{\ell}^{r}\right)$ is a minimal reduction of $I^{r}$. Hence there exist elements $y_{1}, y_{2}, \ldots, y_{s-\ell+1}$ in $I^{r}$ such that $\left(x_{1}^{r}, \ldots, x_{\ell-1}^{r}, y_{1}, \ldots, y_{s-\ell+1}\right)=I^{r}$. Set $P=\left(y_{1}, y_{2}, \ldots, y_{s-\ell+1}\right)$,

$$
e\left(F_{A}(I)\right) \leqslant \mu\left[\left(I^{r}+Q\right) / Q\right]=\mu[(P+Q) / Q] \leqslant \mu(P)=s-\ell(I)+1 .
$$

We get (iv). Theorem 2.3 has been proved.

If ht $I$ is the height of $I$, then ht $(I) \leqslant \ell(I)$. In the case of ht $(I)=\ell(I), I$ is called equimultiple. 
Remark 2.4. Let $I$ be an equimultiple ideal with $h=$ ht $I=\ell(I)>0$. If grade $I=h$ or $A$ is Cohen-Macaulay, then $x_{1}, x_{2}, \ldots, x_{h}$ is a regular sequence, so that

$$
\left(Q: I^{\infty}\right) \cap I^{r}=Q \cap I^{r} \subseteq Q .
$$

Hence by Theorem 2.3 we get $e\left(F_{A}(I)\right)=\mu\left[\left(I^{n}+Q\right) / Q\right]$ for all $n \geq r=r(I)$. If in addition $r(I) \leqslant 1$, then $e\left(F_{A}(I)\right)=\mu[(I+Q) / Q]=\mu(I)-\ell(I)+1$.

Note that $F_{A}(\mathfrak{m})=G(\mathfrak{m})$ is the associated graded ring of $\mathfrak{m}$. It is well-known that $e(G(\mathfrak{m}))=e(A)$ and $\ell(\mathfrak{m})=d=\operatorname{dim} A$. Recall that $v(A)=\mu(\mathfrak{m})$ is the embedding dimension of $A$. As an immediate consequence of Theorem 2.3 we have the following result for the multiplicity of a Noetherian local ring.

Corollary 2.5. Let $(A, \mathfrak{m})$ be a Noetherian local ring of dimension $d>0$ with maximal ideal $\mathfrak{m}$ and infinite residue field $k=A / \mathfrak{m}$. Suppose that $x_{1}, x_{2}, \ldots, x_{d}$ is a weak$(F C)$-sequence in $\mathfrak{m}$ with respect to $\mathfrak{m}$ and $r_{J}(\mathfrak{m})=r$; here $J=\left(x_{1}, x_{2}, \ldots, x_{d}\right)$. Set $Q=\left(x_{1}, x_{2}, \ldots, x_{d-1}\right)$. Then

(i) $e(A)=\mu\left(\frac{\mathfrak{m}^{n}+Q: \mathfrak{m}^{\infty}}{Q: \mathfrak{m}^{\infty}}\right)$ for all $n \geq r$.

(ii) $e(A) \leqslant \mu\left[\left(Q+\mathfrak{m}^{r}\right) / Q\right]$ with equality if and only if

$$
\left(Q: \mathfrak{m}^{\infty}\right) \cap \mathfrak{m}^{r}=\left(Q: \mathfrak{m}^{\infty}\right) \cap \mathfrak{m}^{r+1}(\bmod Q) .
$$

In addition to $r \leqslant 1, e(A) \leqslant v(A)-d+1$.

(iii) $e(A)=\mu\left[\left(Q+\mathfrak{m}^{r}\right) / Q\right]$ if $A$ is Cohen-Macaulay.

Example 2.6. Let $S=k\left[\left[x^{3}, x^{2} y, x y^{2}, y^{3}, z\right]\right]$, where $x, y, z$ are indeterminates over the infinite field $k$. Then $S$ is a Cohen-Macaulay ring with maximal ideal $\mathfrak{M}=\left(x^{3}, x^{2} y, x y^{2}, y^{3}, z\right)$ and $\operatorname{dim} S=3$. Set $J=\left(x^{3}, y^{3}, z\right)$. A direct computation shows that $\mathfrak{M}^{2}=J \mathfrak{M}$. Therefore, $r_{J}(\mathfrak{M}) \leqslant 1$. It can be verified that $v(S)=5$. Hence by Remark 2.4, we get $e(S)=3$.

Remark 2.7. In a Cohen-Macaulay ring $A$ we have $e(A) \geq v(A)-\operatorname{dim} A-1$. On the other hand if $r(\mathfrak{m}) \leqslant 1$, then $e(A) \leqslant v(A)-\operatorname{dim} A-1$ by Corollary 2.5(ii). So we immediately get that $A$ is Cohen-Macaulay with minimal multiplicity; i.e., $e(A)=v(A)-\operatorname{dim} A-1$ if and only if $A$ is Cohen-Macaulay and $r(\mathfrak{m}) \leqslant 1$. This result is proved by Sally in $[\mathrm{Sa}$.

\section{The Cohen-Macaulayness of Fiber cones}

In this section we will discuss the Cohen-Macaulayness of fiber cones.

As remarked in Note 1 , a reduction $J$ of an ideal $I$ is a minimal reduction if and only if $J$ is generated by a weak-(FC)-sequence of the length $\ell(I)$ in $I$ with respect to $(\mathfrak{m}, I)$.

We begin by establishing the following lemma.

Lemma 3.0. Let $I$ be an ideal with analytic spread $\ell(I)=1$ and grade $I=1$. Let $x$ be a regular element in $I$ such that $x A$ is a minimal reduction of $I$. $\operatorname{Set}_{x_{A}}(I)=r$. Then $F_{A}(I)$ is Cohen-Macaulay if and only if $x I^{n-1} \cap \mathfrak{m} I^{n}=x \mathfrak{m} I^{n-1}$ for all $1 \leqslant$ $n \leqslant r$. 
Proof. Since $x$ is a regular element, by Note 2 we have $\mu\left(x I^{n}\right)=\mu\left(I^{n}\right)$. By Lemma $2.2, \mu\left(I^{r}\right)=e\left(F_{A}(I)\right)$. From this it follows that

$$
\begin{aligned}
l\left[F_{A}(I) / x F_{A}(I)\right] & =1+\sum_{1 \leqslant n \leqslant r} l\left[I^{n} / x I^{n-1}+\mathfrak{m} I^{n}\right] \\
& =1+\sum_{1 \leqslant n \leqslant r}\left(l\left[I^{n} / \mathfrak{m} I^{n}\right]-l\left[\left(x I^{n-1}+\mathfrak{m} I^{n}\right) / \mathfrak{m} I^{n}\right]\right) \\
& =1+\sum_{1 \leqslant n \leqslant r}\left(\mu\left(I^{n}\right)-l\left[x I^{n-1} / x I^{n-1} \cap \mathfrak{m} I^{n}\right]\right) \\
& \geq 1+\sum_{1 \leqslant n \leqslant r}\left(\mu\left(I^{n}\right)-l\left[x I^{n-1} / \mathfrak{m} x I^{n-1}\right]\right) \\
& =1+\sum_{1 \leqslant n \leqslant r}\left(\mu\left(I^{n}\right)-\mu\left(x I^{n-1}\right)\right)=\mu\left(I^{r}\right)=e\left(F_{A}(I)\right) .
\end{aligned}
$$

Therefore, $l\left[F_{A}(I) / x F_{A}(I)\right]=e\left(F_{A}(I)\right)$ and hence $F_{A}(I)$ is Cohen-Macaulay if and only if

$$
x I^{n-1} \cap \mathfrak{m} I^{n}=x \mathfrak{m} I^{n-1} \text { for all } 1 \leqslant n \leqslant r .
$$

The main result of this paper is established in the following theorem.

Theorem 3.1. Let $(A, \mathfrak{m})$ be a Noetherian local ring with maximal ideal $\mathfrak{m}$ and infinite residue field $k=A / \mathfrak{m}$. Let $I$ be an ideal with analytic spread $\ell(I)=\ell>0$ and $J$ a minimal reduction of $I$. Suppose that $J$ is generated by $x_{1}, x_{2}, \ldots, x_{\ell}, a$ weak-(FC)-sequence in $I$ with respect to $(\mathfrak{m}, I)$. Set $Q=\left(x_{1}, \ldots, x_{\ell-1}\right)$. Then $F_{A}(I)$ is Cohen-Macaulay if and only if the following conditions are satisfied:

(i) $\left(Q: I^{\infty}\right) \cap I^{n} \subseteq J I^{n-1}\left(\bmod \mathfrak{m} I^{n}\right)$ for all $1 \leqslant n \leqslant r_{J}(I)$.

(ii) $\left(J I^{n-1}+Q: I^{\infty}\right) \cap \mathfrak{m} I^{n}=\mathfrak{m} J I^{n-1}\left(\bmod Q: I^{\infty}\right)$ for all $1 \leqslant n \leqslant r_{J}(I)$.

Proof. Set $A_{\ell-1}=A / Q, I_{\ell-1}=I A_{\ell-1}, A_{\ell-1}^{\prime}=A / Q: I^{\infty}$, and $I_{\ell-1}^{\prime}=I A_{\ell-1}^{\prime}$. Note that $\left(x_{1}, x_{2}, \ldots, x_{\ell}\right)$ is a minimal reduction of $I, \ell\left(I_{\ell-1}^{\prime}\right)=1$. By Proposition 2.1 we have

$$
e\left(F_{A}(I)\right)=e\left(F_{A_{\ell-1}}\left(I_{\ell-1}\right)\right)=e\left(F_{A_{\ell-1}^{\prime}}\left(I_{\ell-1}^{\prime}\right)\right)
$$

and

$$
\begin{aligned}
l\left[F_{A_{\ell-1}^{\prime}}\left(I_{\ell-1}^{\prime}\right) / J F_{A_{\ell-1}^{\prime}}\left(I_{\ell-1}^{\prime}\right)\right] & \leqslant l\left[F_{A_{\ell-1}}\left(I_{\ell-1}\right) / J F_{A_{\ell-1}}\left(I_{\ell-1}\right)\right] \\
& \leqslant l\left[F_{A}(I) / J F_{A}(I)\right] .
\end{aligned}
$$

Note that $F_{A}(I)$ is Cohen-Macaulay if and only if $e\left(F_{A}(I)\right)=l\left[F_{A}(I) / J F_{A}(I)\right]$. This is equivalent to the fact that $F_{A_{\ell-1}^{\prime}}\left(I_{\ell-1}^{\prime}\right)$ is Cohen-Macaulay and

$$
\begin{aligned}
l\left[F_{A_{\ell-1}^{\prime}}\left(I_{\ell-1}^{\prime}\right) / J F_{A_{\ell-1}^{\prime}}\left(I_{\ell-1}^{\prime}\right)\right] & =l\left[F_{A_{\ell-1}}\left(I_{\ell-1}\right) / J F_{A_{\ell-1}}\left(I_{\ell-1}\right)\right] \\
& =l\left[F_{A}(I) / J F_{A}(I)\right] .
\end{aligned}
$$

By Proposition 2.1(iii), (6) is equivalent to

$$
\left(Q: I^{\infty}\right) \cap I^{n} \subseteq J I^{n-1}\left(\bmod \mathfrak{m} I^{n}\right) \text { for all } 1 \leqslant n \leqslant r_{J}(I) .
$$

It is clear that grade $I_{\ell-1}^{\prime}=1=\ell\left(I_{\ell-1}^{\prime}\right)$. Hence by Lemma 3.0, $F_{A_{\ell-1}^{\prime}}\left(I_{\ell-1}^{\prime}\right)$ is Cohen-Macaulay if and only if

$$
\left(J I^{n-1}+Q: I^{\infty}\right) \cap\left(\mathfrak{m} I^{n}+Q: I^{\infty}\right)=\mathfrak{m} J I^{n-1}\left(\bmod Q: I^{\infty}\right)
$$


for all $1 \leqslant n \leqslant r_{J}(I)$. It can be verified that this condition is equivalent to

$$
\left(J I^{n-1}+Q: I^{\infty}\right) \cap \mathfrak{m} I^{n}=\mathfrak{m} J I^{n-1}\left(\bmod Q: I^{\infty}\right) \text { for all } 1 \leqslant n \leqslant r_{J}(I) .
$$

Therefore, $F_{A}(I)$ is Cohen-Macaulay if and only if the following conditions are satisfied:

$$
\begin{aligned}
\left(Q: I^{\infty}\right) \cap I^{n} & \subseteq J I^{n-1}\left(\bmod \mathfrak{m} I^{n}\right) \\
\text { and }\left(J I^{n-1}+Q: I^{\infty}\right) \cap \mathfrak{m} I^{n} & =\mathfrak{m} J I^{n-1}\left(\bmod Q: I^{\infty}\right)
\end{aligned}
$$

for all $1 \leqslant n \leqslant r_{J}(I)$. This completes the proof of Theorem 3.1.

We now examine how particular cases of Theorem 3.1 can be treated. In the case of $r(I) \leqslant 1$, by different approaches, Huneke and Sally [H-S] proved that if $A$ is Cohen-Macalay and $I$ is $m$-primary, then $F_{A}(I)$ is Cohen-Macaulay. Shah Sh1 extended this result and showed that $F_{A}(I)$ is Cohen-Macaulay if $I$ is an equimultiple ideal with grade $I=$ ht $I$. These results are particular cases of the following proposition:

Proposition 3.2. Let $(A, \mathfrak{m})$ be a Noetherian local ring with maximal ideal $\mathfrak{m}$ and infinite residue field $k=A / \mathfrak{m}$. Let $I$ be an ideal with analytic spread $\ell(I)=\ell>0$ and $J$ a minimal reduction of $I$. Suppose that $J$ is generated by $x_{1}, x_{2}, \ldots, x_{\ell}, a$ weak-(FC)-sequence in $I$ with respect to $(\mathfrak{m}, I)$ and $r_{J}(I) \leqslant 1$. Set $Q=\left(x_{1}, \ldots\right.$, $\left.x_{\ell-1}\right)$. Then $F_{A}(I)$ is Cohen-Macaulay if $Q: I^{\infty} \subseteq J$.

Proof. Since $r_{J}(I) \leqslant 1$, by Theorem 3.1 it follows that $F_{A}(I)$ is Cohen-Macaulay if and only if

$$
\left(Q: I^{\infty}\right) \cap I \subseteq J(\bmod \mathfrak{m} I)
$$

and $\left(J+Q: I^{\infty}\right) \cap \mathfrak{m} I=\mathfrak{m} J\left(\bmod Q: I^{\infty}\right)$. But $Q: I^{\infty} \subseteq J$, the first equation is obvious, and the second equation is equivalent to $J \cap \mathfrak{m} I=\mathfrak{m} J\left(\bmod Q: I^{\infty}\right)$. To obtain this equation, it is enough to show $J \cap \mathfrak{m} I=\mathfrak{m} J$. Since $J$ is a minimal reduction of $I$, there exist elements $y_{1}, \ldots, y_{m}$ in $I$ such that $x_{1}, x_{2}, \ldots, x_{\ell}, y_{1}, \ldots, y_{m}$ is a minimal base of $I$. Now assume that $x=a_{1} x_{1}+\cdots+a_{\ell} x_{\ell} \in J \cap \mathfrak{m} I$,

$$
x=b_{1} x_{1}+\cdots+b_{\ell} x_{\ell}+c_{1} y_{1}+\cdots+c_{m} y_{m},
$$

and $b_{i} \in \mathfrak{m}$ for $1 \leqslant i \leqslant \ell$ and $c_{j} \in \mathfrak{m}$ for $1 \leqslant j \leqslant m$. Therefore,

$$
\left(a_{1}-b_{1}\right) x_{1}+\cdots+\left(a_{\ell}-b_{\ell}\right) x_{\ell}+c_{1} y_{1}+\cdots+c_{m} y_{m}=0
$$

Since $x_{1}, x_{2}, \ldots, x_{\ell}, y_{1}, \ldots, y_{m}$ is a minimal base of $I, a_{i}-b_{i} \in \mathfrak{m}$ for $1 \leqslant i \leqslant \ell$. Hence $a_{i} \in \mathfrak{m}$ for $1 \leqslant i \leqslant \ell$. Thus, $J \cap \mathfrak{m} I=\mathfrak{m} J$. We get the proof of Proposition 3.2.

Remark 3.3. Note that if $r(I) \leqslant 1$ and $I$ is an equimultiple ideal with grade $I=$ ht $I=h>0$ and $x_{1}, x_{2}, \ldots, x_{h}$ is a weak-(FC)-sequence in $I$, then $x_{1}, x_{2}, \ldots, x_{h}$ is a regular sequence. Hence

$$
\left(x_{1}, \ldots, x_{h-1}\right): I^{\infty}=\left(x_{1}, \ldots, x_{h-1}\right) .
$$

By Proposition 3.2, we immediately get the results of Huneke-Sally and Shah as in [op. cit.].

Remark 3.4. Corso-Polini-Vasconcelos C-P-V1], Corso-Polini [C-P], and CorsoHuneke-Vasconcelos [C-H-V] proved that if $\mathrm{A}$ is Cohen-Macaulay with $e(A)>1$ and $J$ is a parameter ideal of $A$, then $I^{2}=I J$, where $I=J: \mathfrak{m}$. Therefore, $r(I) \leqslant 1$. Hence we have $F_{A}(I)$ Cohen-Macaulay by Remark 3.3 and $e\left(F_{A}(I)\right)=\mu(I)-d+1$ by Remark 2.7 . 
In the case in which a minimal reduction $J$ of $I$ is generated by a regular sequence and $J \cap I^{n}=J I^{n-1}$ for all $n \leqslant r_{J}(I)$, Cortadellas and Zarzuela C-Z proved that $F_{A}(I)$ is Cohen-Macaulay if and only if $J \cap \mathfrak{m} I^{n}=J \mathfrak{m} I^{n-1}$ for all $n \leqslant r_{J}(I)$.

As an immediate consequence of Theorem 3.1 we get a result that seems to account well for the theorem of Cortadellas-Zarzuela [op. cit.].

Corollary 3.5. Let $I$ be an equimultiple ideal of height ht $I=h>0$ and grade $I=$ $h$; $J$ is a minimal reduction of $I$. Suppose that $J$ is generated by $x_{1}, x_{2}, \ldots, x_{h}, a$ weak-(FC)-sequence in $I$ with respect to $(\mathfrak{m}, I)$. Set $Q=\left(x_{1}, \ldots, x_{h-1}\right)$. Then $F_{A}(I)$ is Cohen-Macaulay if and only if the following conditions are satisfied:

(i) $Q \cap I^{n} \subseteq J I^{n-1}\left(\bmod \mathfrak{m} I^{n}\right)$ for all $1 \leqslant n \leqslant r_{J}(I)$.

(ii) $\left(J I^{n-1}+Q\right) \cap \mathfrak{m} I^{n}=\mathfrak{m} J I^{n-1}(\bmod Q)$ for all $1 \leqslant n \leqslant r_{J}(I)$.

If $J \cap I^{n}=J I^{n-1}$ for all $n \leqslant r_{J}(I)$, then condition (i) is always true and

$$
\begin{aligned}
\left(J I^{n-1}+Q\right) \cap \mathfrak{m} I^{n} & =\left(J I^{n-1}+Q\right) \cap I^{n} \cap \mathfrak{m} I^{n} \\
& =\left(J I^{n-1}+Q \cap I^{n}\right) \cap \mathfrak{m} I^{n} \\
& =J I^{n-1} \cap \mathfrak{m} I^{n} \\
& =J \cap I^{n} \cap \mathfrak{m} I^{n}=J \cap \mathfrak{m} I^{n} .
\end{aligned}
$$

Thus, if $J \cap \mathfrak{m} I^{n}=J \mathfrak{m} I^{n-1}$ for all $n \leqslant r_{J}(I)$, then condition (ii) is satisfied. This recovers the theorem of Cortadellas-Zarzuela [op. cit.].

By Corollary 2.5(ii), $e(A) \leqslant v(A)-d+1$ if $r(I) \leqslant 1$. In the case that $A$ is CohenMacaulay it is well-known that $e(A) \geq v(A)-d+1$. If $e(A)=v(A)-d+1$, it is said that $A$ has minimal multiplicity. We know by Sally $\mathrm{Sa}$ that $A$ is Cohen-Macaulay with minimal multiplicity if and only if $A$ is Cohen-Macaulay and $r(\mathfrak{m}) \leqslant 1$.

In answer to the question as to when the fiber cone is Cohen-Macaulay with minimal multiplicity, we have the following theorem.

Theorem 3.6. Let $(A, \mathfrak{m})$ be a Noetherian local ring with maximal ideal $\mathfrak{m}$ and infinite residue field $k=A / \mathfrak{m}$. Let $I$ be an ideal with analytic spread $\ell(I)=\ell>0$ and $J$ a minimal reduction of $I$. Suppose that $J$ is generated by $x_{1}, x_{2}, \ldots, x_{\ell}$, a weak-(FC)-sequence in $I$ with respect to $(\mathfrak{m}, I)$. Set $Q=\left(x_{1}, \ldots, x_{\ell-1}\right)$. Then $F_{A}(I)$ is Cohen-Macaulay with minimal multiplicity if and only if $r_{J}(I) \leqslant 1$ and the following conditions are satisfied:

(i) $\left(Q: I^{\infty}\right) \cap I \subseteq J(\bmod \mathfrak{m} I)$.

(ii) $\left(J+Q: I^{\infty}\right) \cap \mathfrak{m} I=\mathfrak{m} J\left(\bmod Q: I^{\infty}\right)$.

Proof. Note that $v\left(F_{A}(I)\right)=\mu(I)$. Therefore, $F_{A}(I)$ has minimal multiplicity if $e\left(F_{A}(I)\right)=\mu(I)-\ell(I)+1$. Hence $F_{A}(I)$ is Cohen-Macaulay with minimal multiplicity if and only if $F_{A}(I)$ is Cohen-Macaulay and

$l\left[F_{A}(I) / J F_{A}(I)\right]=\mu(I)-\ell(I)+1+\sum_{2 \leqslant n \leqslant r} l\left[I^{n} /\left(J I^{n-1}+\mathfrak{m} I^{n}\right)\right]=\mu(I)-\ell(I)+1$.

This is equivalent to $r_{J}(I) \leqslant 1$ and $F_{A}(I)$ is Cohen-Macaulay. Hence the proof is complete by Theorem 3.1.

We recall that $F_{A}(\mathfrak{m})=G(\mathfrak{m})$ and $e(A)=e(G(\mathfrak{m})) ; v(A)=v(G(\mathfrak{m}))$. Hence $G(\mathfrak{m})$ has minimal multiplicity if and only if $A$ has minimal multiplicity. If $I=\mathfrak{m}$ and $r_{J}(I) \leqslant 1$, then $\mathfrak{m} I=\mathfrak{m}^{2} \subseteq J$, and hence the condition $\left(Q: I^{\infty}\right) \cap I \subseteq$ $J(\bmod \mathfrak{m} I)$ is equivalent to $Q: \mathfrak{m}^{\infty} \subseteq J$. Then as an immediate consequence of Proposition 3.2 and Theorem 3.6, we have: 
Corollary 3.7. Let $(A, \mathfrak{m})$ be a Noetherian local ring with maximal ideal $\mathfrak{m}$ and infinite residue field $k=A / \mathfrak{m}$. Suppose that $J$ is generated by $x_{1}, x_{2}, \ldots, x_{d}$, a weak$(F C)$-sequence in $\mathfrak{m}$ with respect to $\mathfrak{m}$. Set $Q=\left(x_{1}, \ldots, x_{d-1}\right)$. Then $G(\mathfrak{m})$ is CohenMacaulay with minimal multiplicity if and only if $r_{J}(\mathfrak{m}) \leqslant 1$ and $Q: \mathfrak{m}^{\infty} \subseteq J$.

Remark 3.8. Denote by $R(\mathfrak{m})$ the Rees algebra of $\mathfrak{m}$. By Goto and Shimoda in G-S], if $A$ is Cohen-Macaulay, $R(\mathfrak{m})$ is Cohen-Macaulay if and only if $G(\mathfrak{m})$ is Cohen-Macaulay and $r(\mathfrak{m}) \leqslant \operatorname{dim} A-1$. Now if $A$ is Cohen-Macaulay of $\operatorname{dim} A>1$ and $r(\mathfrak{m}) \leqslant 1$, then by Corollary $3.7 G(\mathfrak{m})$ is Cohen-Macaulay. Hence $R(\mathfrak{m})$ is also Cohen-Macaulay.

Example 3.9. Let $S=k\left[\left[x^{3}, x^{2} y, x y^{2}, y^{3}, z\right]\right]$, where $x, y, z$ are indeterminates over the infinite field $k$. Then $S$ is Cohen-Macaulay with maximal ideal $\mathfrak{M}=$ $\left(x^{3}, x^{2} y, x y^{2}, y^{3}, z\right)$ and $\operatorname{dim} S=3$. Set $J=\left(x^{3}, y^{3}, z\right), \mathfrak{M}^{2}=J \mathfrak{M}$. So $r(\mathfrak{M})=1$. Hence $R(\mathfrak{M})$ is Cohen-Macaulay.

From Theorem 3.6 and Proposition 3.2, we also immediately get the following result.

Corollary 3.10. Let $I$ be an equimultiple ideal of ht $I=h>0$ and grade $I=h$. Then $F_{A}(I)$ is Cohen-Macaulay with minimal multiplicity if and only if $r(I) \leqslant 1$.

\section{ACKNOWLEDGEMENT}

The author is indebted to the referee for valuable comments and suggestions.

\section{REFERENCES}

[C-G-P-U] A. Corso, L. Ghezzi, C. Polini, B. Ulrich, Cohen-Macaulayness of special fiber rings, Comm. Algebra 31(2003), 3713-3734. MR2007381 (2004k:13006)

[C-H-V] A. Corso, C. Huneke, W. V. Vasconcelos, On the integral closure of ideals, Manuscripta Math. 95(1998), 331-347. MR1612078 (99b:13010)

[C-P] A. Corso, C. Polini, Links of prime ideals and their Rees algebras, J. Algebra 178(1995), 224-238. MR1358263 (96i:13006)

[C-P-V1] A. Corso, C. Polini, W. V. Vasconcelos, Links of prime ideals, Math. Proc. Camb. Phil. Soc. 115(1994), 431-436. MR1269930 (95d:13001)

[C-P-V2] A. Corso, C. Polini, W. V. Vasconcelos, Multiplicity of the special fiber ring of blowups, Math. Proc. Camb. Phil. Soc. 140(2006), 207-219. MR2212275 (2006k:13052)

[C-Z] T. Cortadellas, S. Zarzuela, On the depth of the fiber cone of filtrations, J. Algebra 198(1997), 428-445. MR1489906 (98k:13005)

[Co-Z] T. Cortadellas, S. Zarzuela, On the structure of the fiber cone of ideals with analytic spread one, arXiv: math.AC/0603042 v1 2 Mar 2006.

[D-G-H] M. D'Anna, A. Guerrieri, W. Heinzer, Ideals having one-dimensional fiber cone, Lecture Notes in Pure and Appl. Math. 220, Dekker, 2001, 155-170. MR1836599 (2002c:13006)

[D-R-V] C. D’Cruz, K. N. Raghavan, J. K. Verma, Cohen-Macaulay fiber cones, Commutative algebra, algebraic geometry and computational methods (Hanoi, 1996), 233-246, Springer, Singapore, 1999. MR.1714861 (2000i:13003)

[G-S] S. Goto, Y. Shimoda, On the Rees algebras of Cohen-Macaulay local rings, Lect. Notes in Pure and Appl. Math. 68, Dekker, 1979, 201-231. MR655805 (84a:13021)

$[\mathrm{H}-\mathrm{H}] \quad$ R. Hübl, C. Huneke, Fiber cones and the integral closure of ideals, Collect. Math. 52(2001), 85-100. MR1833088 (2002h:13007)

[H-S] C. Huneke, J. D. Sally, Birational extensions in dimension two and integrally closed ideals, J. Algebra 115(1988), 481-500. MR943272 (89e:13025)

[J-V-S] A. V. Jayanthan, J. K. Verma, B. Singh, Hilbert coefficients and depth of form rings, Comm. Algebra 32(2004), 1445-1452. MR2100366 (2005g:13008)

[J-V-V1] A. V. Jayanthan, J. K. Verma, Hilbert coefficients and depth of fiber cones, J. Pure Appl. Algebra 201(2005), 97-115. MR2158749(2006d:13024) 
[J-V2] A. V. Jayanthan, J. K. Verma, Fiber cones of ideals with almost minimal multiplicity, Nagoya Math. J. 177(2005), 155-179. MR2124550(2006h:13007)

[J-P-V] A. V. Jayanthan, T. J. Puthenpurakal, J. K. Verma, On fiber cones of m-primary ideals, Canad. J. Math. 59(2007), 109-126. MR2289420

[N-R] D. G. Northcott and D. Rees, Reduction of ideals in local rings, Proc. Cambridge Phil. Soc. 50(1954), 145-158. MR0059889(15:596a)

[Re] D. Rees, Generalizations of reductions and mixed multiplicities, J. London Math. Soc. 29(1984), 397-414. MR754926 (86e:13023)

[Sa] J. D. Sally, On the associated graded ring of a local Cohen-Macaulay ring, J. Math. Kyoto 17(1977), 19-21. MR0450259 (56:8555)

[Sh1] K. Shah, On the Cohen-Macaulayness of the fiber cone of an ideal, J. Algebra 143(1991), 156-172. MR1128652 (92k:13014)

[Sh2] K. Shah, On equimultiple ideals, Math. Z. 215(1994), 13-24. MR1254811 (95j:13021)

[Vi1] D. Q. Viet, Mixed multiplicities of arbitrary ideals in local rings, Comm. Algebra 28(8)(2000), 3803-3821. MR1767591(2001f:13036)

[Vi2] D. Q. Viet, On some properties of $(F C)$-sequences of ideals in local rings, Proc. Amer. Math. Soc. 131(1)(2003), 45-53. MR.1929022 (2003i:13033)

[Vi3] D. Q. Viet, Sequences determining mixed multiplicities and reductions of ideals, Comm. Algebra 31(10)(2003), 5047-5069. MR1998043 (2004f:13030)

[Vi4] D. Q. Viet, The multiplicity and the Cohen-Macaulayness of extended Rees algebras of equimultiple ideals, J. Pure Appl. Algebra 205(2006), 498-509. MR2210217 (2006k:13007)

Department of Mathematics, Hanoi University of Education, Xuan Thuy Street, HANOI, VIETNAM

E-mail address: duongquocviet@fmail.vnn.vn 